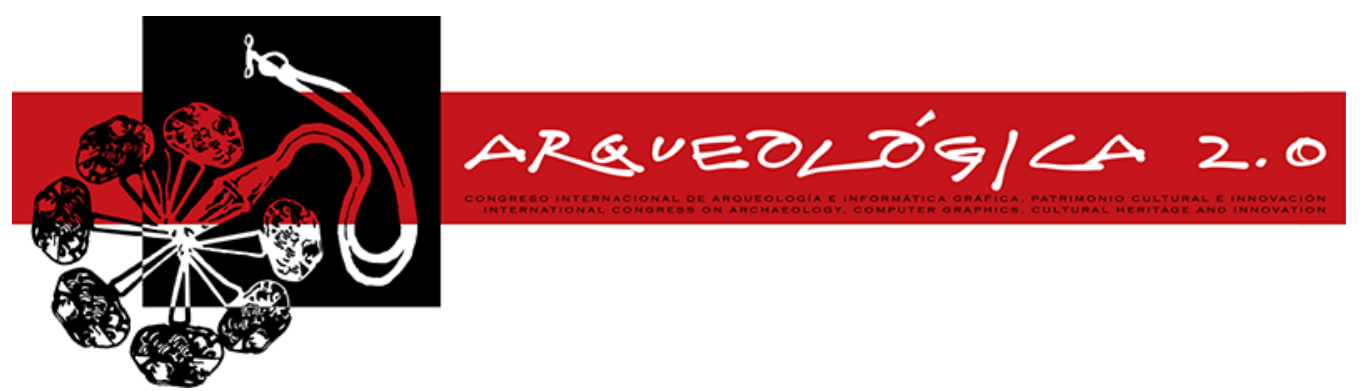

Proceedings of the $8^{\text {th }}$ International Congress

on Archaeology,

Computer Graphics,

Cultural Heritage and Innovation

'ARQUEOLÓGICA 2.0'

in Valencia (Spain),

Sept. 5-7, 2016

DOI: $\underline{\text { http://dx.doi.org/10.4995/arqueologica8.2016.3562 }}$

\title{
MODELIZACIÓN Y SIMULACION DE LAS POSIBLES POSICIONES DE LAS ATALAYAS DE LA FORTALEZA DE LA MOTA EN ALCALA LA REAL, MEDIANTE TECNICAS DE ANALISIS VISUAL
}

\section{MODELING AND SIMULATION OF POSSIBLE POSITIONS OF THE WATCHTOWER NETWORK OF THE MOTA FORTRESS IN ALCALA LA REAL, USING VISUAL ANALYSIS TECHNIQUES}

\author{
Francisco J. Rosales ${ }^{*}$ José Luis Pérez-García, Carlos Colomo, José M. Gómez-López, Manuel A. \\ Ureña
}

Departamento de Ingeniería Cartográfica, Geodésica y Fotogrametría, Escuela Politécnica Superior de Jaén, Campus de las Lagunillas, Universidad de Jaén s/n, 23071 Jaén, España. fira0004@red.ujaen.es, ilperez@ujaen.es; cmcj0002@red.ujaen.es; iglopez@ujaen.es; maurena@ujaen,es

\begin{abstract}
:
The cultural heritage plays a very important role in the Smart management of an area, and geospatial technologies are a perfect tool for the heritage knowledge, management and analysis. Photogrammetry, UAV systems and geographic information systems, can help in cataloguing the cultural heritage of a city. The main turistic value for Alcalá la Real, is the Monumental Group of the Fortress of La Mota and their related monuments.

As the principal objetive of the Project, an analysis and the calculation of the optimal location of the watchtower network is made. To achieve this, on the one hand photogrammetry technics are used to get the geometric information of the studied area. On the other hand, photogrammetry through UAV systems is used to obtain the 3D model of one of the watchtowers, which is better preserved than others. Finally, programming tools are applied over GIS for the analysis and calculation of the optimal location of this watchtower network.
\end{abstract}

Key words: virtual archaeology, visibility, GIS, cultural heritage, watchtower

\section{Resumen:}

El patrimonio cultural juega un papel importante en la gestión inteligente de un territorio, y las técnicas geoespaciales son una perfecta herramienta para el conocimiento, gestión y análisis de dicho patrimonio. Elementos como la fotogrametría, los sistemas UAV y los sistemas de información geográfica, pueden contribuir en gran medida a la catalogación y puesta en valor del patrimonio de una ciudad. Alcalá la Real tiene al Conjunto Monumental de la Fortaleza de la Mota y elementos relacionados como motor principal del atractivo turístico de la ciudad. Motivo por el que se plantea utilizar diversas técnicas geoespaciales para el estudio y análisis de parte de ese patrimonio.

Concretamente, como objetivo principal del trabajo, se plantea el análisis y la determinación de la ubicación óptima de la red de atalayas. Para ello se utilizan técnicas fotogramétricas para obtener la información geométrica de la zona de estudio a partir de imágenes de un vuelo fotogramétrico. Por otro lado, para el conocimiento de las características de las atalayas, se realiza el modelado de una de las mejor conservadas, mediante fotogrametría con sistemas UAV. Finalmente, se utilizan herramientas de programación sobre sistemas de información geográfica para el análisis y la determinación de la ubicación óptima de la red de atalayas.

Palabras clave: arqueología virtual, visibilidad, SIG, patrimonio cultural, atalaya

\section{Introducción}

El patrimonio cultural juega un papel importante en la gestión inteligente de un territorio, siendo las técnicas geoespaciales una perfecta herramienta para el conocimiento, gestión y análisis de dicho patrimonio (Torres et al. 2012). El uso de técnicas como la fotogrametría, los sistemas UAV o los SIG, contribuyen en gran medida a la catalogación y puesta en valor del patrimonio de una zona (Zamora et al. 2012.). 
El objetivo principal del presente trabajo es el análisis y determinación de la ubicación óptima de la red de atalayas, a partir de procesos de simulación en un entorno SIG. Para ello, se hace uso de datos digitales de carácter geográfico facilitados por el Centro Nacional de Información Geográfica (CNIG), para la elaboración de una cartografía base, así como, datos capturados por sistemas UAVs para la modelización y parametrización de las atalayas que aún se conservan.

\subsection{Contexto Histórico}

Alcalá la Real fue un enclave de gran importancia estratégica en el sur de la Península Ibérica. Este hecho, y en especial el carácter de frontera que tuvo en el pasado han determinado el devenir histórico de la ciudad. Está presidida por el Conjunto Monumental de la Fortaleza de la Mota, declarada de Bien Cultural de Andalucía junto a sus murallas, Torre de Alcazaba y su red de atalayas.

La estrategia militar en la Edad Media daba gran importancia a las torres vigía. Estas servían como posiciones avanzadas que informaban de los movimientos del enemigo y de sus posibles ataques.

En torno a Alcalá la Real se configuró un amplio dispositivo de atalayas cristianas y musulmanas, como llave del sistema fronterizo en la Baja Edad Media, poniendo en contacto castillos y ciudades como Alcaudete, Moclín y la propia Alhambra. Hoy día, muchas de esas atalayas han desaparecido o se han visto deterioradas, la disposición de las mejor conservadas se muestra en la Figura 1.

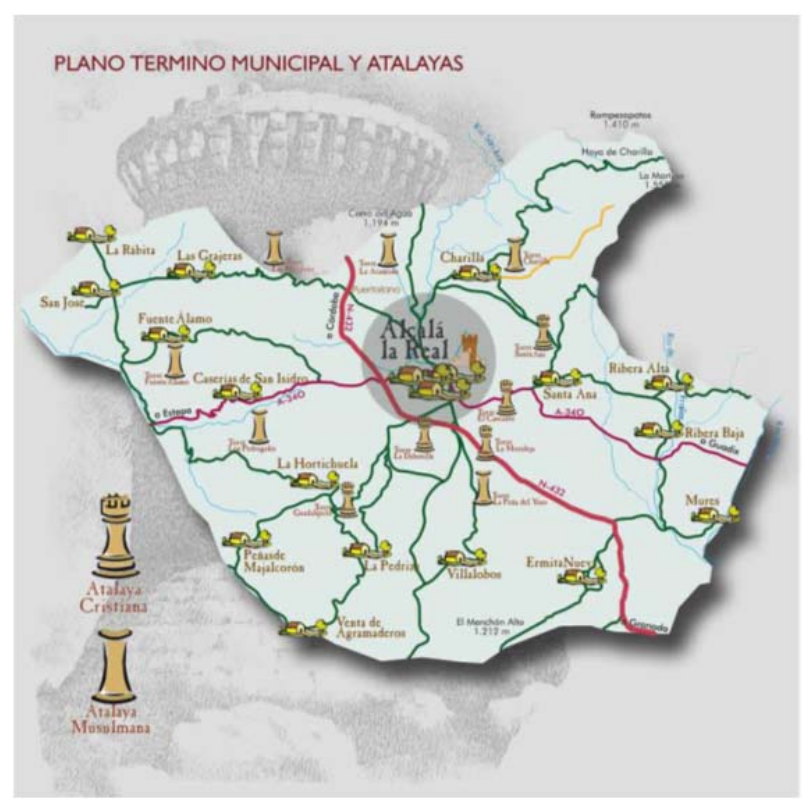

Figura 1: Red de atalayas existentes

\subsection{Análisis de visibilidad}

De entre la gran cantidad de las posibilidades de análisis que presentan los Sistemas de Información Geográfica, las capacidades de análisis de visibilidad abren un amplio campo de posibilidades en trabajos de arqueología virtual. La idea que presentan estos análisis son la de evaluar aquellos lugares que son visibles desde otros (Villanueva 2012).
Dada una superficie del terreno y un punto de vista, el problema clásico de la visibilidad es detectar la porción de terreno visible desde dicho punto, esto es, la cuenca visual. Diversas aéreas de conocimiento utilizan esta técnica como herramienta de análisis.

La morfología de una superficie de terreno afecta en gran medida qué partes de la superficie puede ver un observador desde en un punto determinado. Estos análisis, gracias a la alta capacidad de procesamiento de los ordenadores actuales, se postulan como herramientas imprescindibles en otras áreas de estudio y son utilizados de manera extensa en áreas de estudio como el planeamiento territorial, geomarketing.

El objetivo es determinar la visibilidad de punto a punto a lo largo de una línea de visión determinada o a través de una superficie en una cuenta visual.

\subsubsection{Cuencas Visuales}

Los análisis de cuencas visuales pretenden identificar las zonas visibles que pueden observarse desde una 0 más ubicaciones de observación. Este tipo de análisis requiere de una serie de datos de entrada, siendo estos:

- Modelo Digital del Terreno: La visibilidad es calculada atendiendo a la geometría del terreno, de manera que este dato es fundamental en el estudio, siendo a su vez un factor muy importante, dado que su calidad determinará en gran medida la calidad de los resultados obtenidos.

- Punto/s Observador: ubicación de la cual se desea saber su cuenca visual, puede ser uno o varios puntos. Pudiendo encontrarse estos observadores sobre el terreno, o sobre algún elemento que le permita situarse a cierta altura del mismo.

Con estos datos, el proceso de cálculo de la cuenca visual se desarrolla a partir de anillos concéntricos desde el punto observador.

El procedimiento es en realidad bastante simple computacionalmente, y se parece en esencia al cálculo de la proximidad efectiva. Podemos imaginarlo como una serie de "olas de distancia" que se originan en el punto considerado y se van radiando hacia afuera, como si hubiéramos arrojado una piedra en un estanque (véase la Figura 2). A medida que las olas se propagan, se calculan la distancia desde la celda de observación inicial (denominada celda "run") y la diferencia de elevación con cada una de las celdas que forman el anillo concéntrico de una ola (denominadas celdas "rise"). Si el coeficiente rise/run es mayor que cualquiera de los coeficientes de los anillos concéntricos previos, la celda en cuestión para la que estemos analizando el valor será catalogada como vista. Si por el contrario dicho coeficiente es menor, la celda pasará a ser marcada como no vista.

\subsubsection{Líneas de visión 3D}

Mediante este análisis obtenemos una línea entre dos puntos que muestra las partes de la superficie a lo largo de dicha línea que son visibles o que están ocultas para el observador. Determinando así si un punto es visible desde otro punto. Si el terreno oculta el punto de destino, puede ver dónde se encuentra la obstrucción y qué más puede verse o está oculto a lo largo de la línea 
de visión. En la Figura 3, los segmentos visibles se muestran en verde y los segmentos ocultos se muestran en rojo. Un punto negro al inicio de la línea representa la ubicación del observador. Un punto azul representa el punto de obstrucción desde el observador al destino. Un punto rojo al final de la línea representa la ubicación de destino.

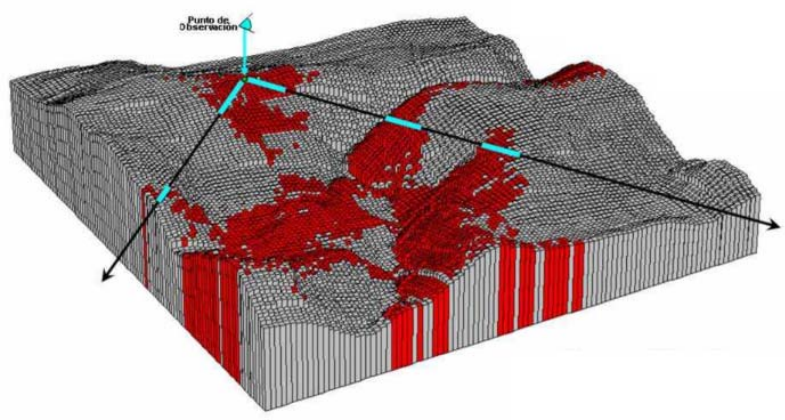

Figura 2: Cuenca Visual

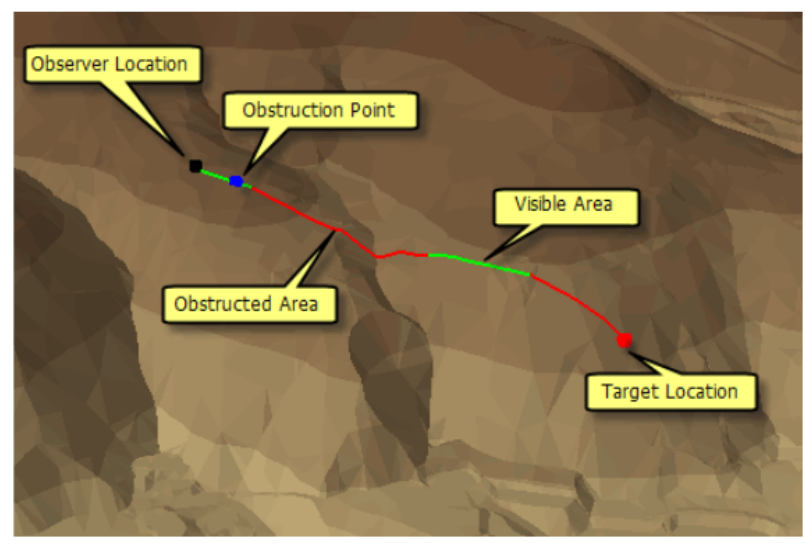

Figura 3: Líneas de visión

\subsection{Programación entorno SIG}

Las herramientas propias del software SIG nos permiten realizar tareas de manera básica e intuitiva por medio de la interfaz propia de cada herramienta. Para realizar análisis más complejos con flujos de trabajo, procesado de datos por lotes, automatización y optimización de estos análisis, se puede hacer uso de lenguajes de programación para la creación de scripts que contengan las órdenes a llevar a cabo por el software SIG. El software utilizado ArcGIS, contiene el módulo ArcPy que permite la creación y ejecución de estos scripts.

A modo de resumen se presenta en la Figura 4 un diagrama con el esquema general del proceso de simulación de la red de atalayas en el entorno de la fortaleza de la Mota. Este esquema general tiene sus particularidades a la hora de calcular la posición de las distintas torres en función del método de simulación seleccionado.

El proceso se realiza de manera totalmente automatizada, se ejecuta externamente al software GIS ejecutando el script diseñado. Solo requiere de los datos de entrada correspondientes, en este caso el modelo digital del terreno y el punto observador principal, es decir, la posición geográfica de la Fortaleza de la Mota.

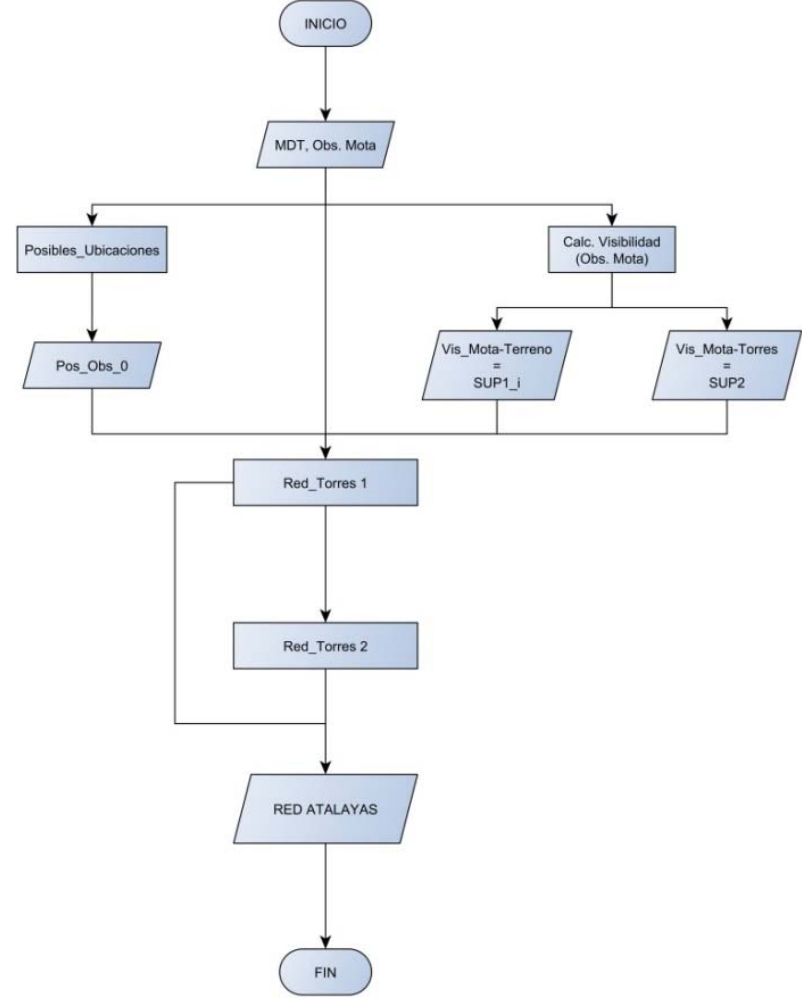

Figura 4: Flujo de Trabajo

\section{Datos de entrada}

\subsection{Modelo Digital del Terreno}

Para la realización de los estudios de visibilidad sobre el Sistema de Información Geográfica, es necesario disponer de un Modelo Digital del Terreno que sirva de base para los diferentes estudios, para ello se utiliza información libre descargada del Centro Nacional de Información Geográfica del IGN, en concreto el "MDT05", que contienen el modelo digital del terreno con paso de malla de $5 \mathrm{~m}$, con la misma distribución de hojas que el MTN50 (véase la Figura 5).

El formato archivo utilizado es ARC-GRID de ESRI. EI sistema geodésico de referencia de la información es ETRS89 y la proyección UTM en huso 30 para este caso.

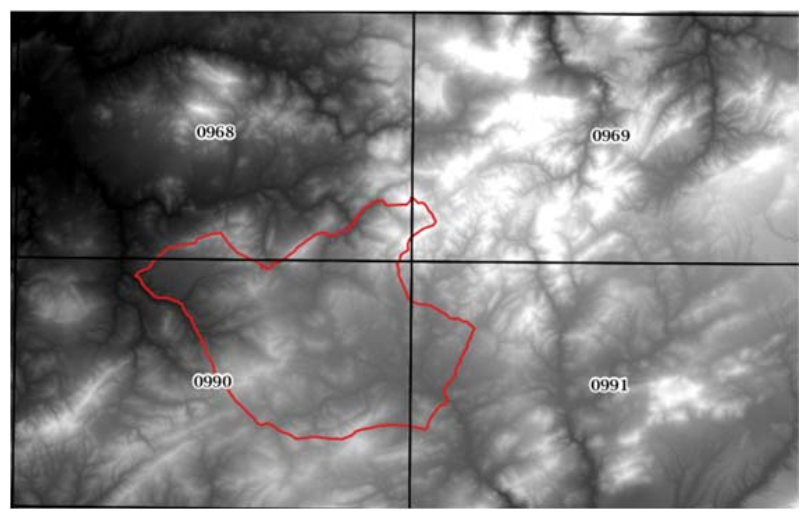

Figura 5: Hojas de MDT utilizadas 


\subsection{Posición de las atalayas existentes}

La posición teórica de las atalayas existentes se obtiene a partir de información proporcionada por el Excmo. Ayuntamiento de Alcalá la Real, el cual proporciona información en formato CAD de la situación de las atalayas que hoy día se encuentran en mejor o peor estado de conservación.

\subsection{Modelo 3D de atalaya}

Con el fin de conocer las características geométricas de las atalayas y de realizar una virtualización y extraer información geométrica de las mismas, se realiza la obtención del modelo 3D de dos de estas atalayas mediante fotogrametría con UAV.

La elección de las atalayas a modelizar se realiza teniendo en cuenta el estado de conservación de las existentes. En la Figura 6, se puede observar una de las seleccionadas denominada "Torre de la Moraleja".

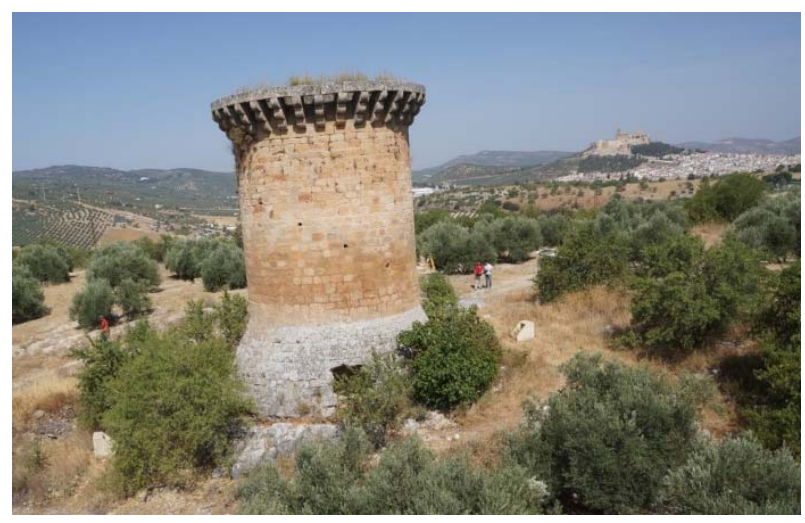

Figura 6: Torre de la Moraleja

Para la obtención del modelo se realizan varios vuelos (cenitales y convergentes) mediante UAV con cámara compacta no métrica. A partir de técnicas fotogramétricas se realiza la virtualización de la atalaya, obteniendo un modelo 3D de la misma, utilizando el software Photoscan.

\section{Propuesta metodológica}

\subsection{Consideraciones previas}

Para la realización de los diferentes estudios, se deben tener en cuenta varios parámetros que afectan al análisis de visibilidad, los cuales se deben establecer a priori. Estos son los siguientes:

\subsubsection{Parametrización de atalaya}

Viene definida tras el conocimiento de las características geométricas de la atalaya objeto de estudio a partir del modelo 3D obtenido mediante fotogrametría con UAV. A efectos de análisis se establece como altura estándar de las atalayas el valor de $10 \mathrm{~m}$ (véase la Figura 7).

\subsubsection{Rango de visibilidad}

Se debe establecer el parámetro de límite máximo de visibilidad, puesto que si no se especifica dicho valor, el software lo considera por defecto como infinito. El límite de visibilidad del ojo humano es difícil de establecer, puesto que depende de condiciones externas, como meteorología, elementos de ayuda a la visión, diferentes señales de alerta. Debido a esto se realiza un análisis de la distancia media entre la Fortaleza y las atalayas, ya que se conoce que la Fortaleza no tiene conectividad visual con todas ellas, se establece como límite máximo de visibilidad una aproximación a la media de estas distancias. A efectos de realización de los estudios, se establece un límite máximo de visibilidad de $6000 \mathrm{~m}$.

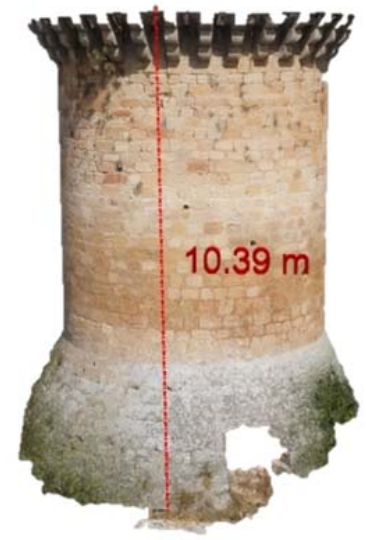

Figura 7: Modelo con dimensiones de atalaya

\subsection{Determinación de posibles ubicaciones}

Las posibles ubicaciones de la red de atalayas dependen directamente del modelo digital del terreno. En principio, se podría plantear que cualquier posición de este modelo digital podría ser una potencial situación de una atalaya. Sin embargo, dado que lo más lógico es que se situaran en lugares localmente elevados, y para disminuir los tiempos de procesamiento, realizando una búsqueda más optimizada, se lleva a cabo una extracción y filtrado de máximos locales en dicho modelo.

Los máximos locales se definen como puntos, que son máximos en relación a sus puntos vecinos analizados. Con este análisis se consigue reducir el número de posibles ubicaciones para atalayas, de un total de 20 millones iniciales a 500 puntos destacados (véase Figura 8).

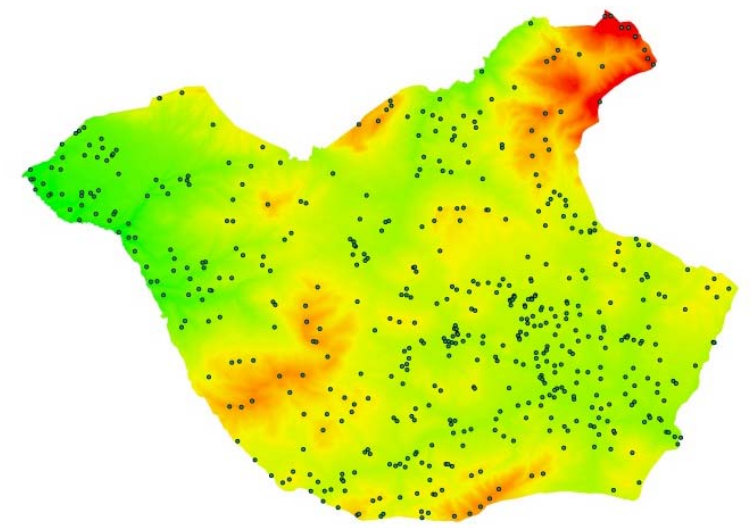

Figura 8: MDT con posibles ubicaciones

Una vez se realiza este proceso, se tendrían todos los datos de entrada para las simulaciones, los cuales son:

- Información geográfica de la Fortaleza de la Mota 
- Modelo digital del terreno.

- Máximos locales como ubicaciones posibles de la red de atalayas.

\subsection{Simulaciones}

Este apartado contiene la descripción de los procesos seguidos para la determinación de la ubicación óptima de la red de atalayas de la Fortaleza de la Mota. El cálculo de la ubicación óptima viene precedido de la imposición de algunos factores que determinan el modo de realizar el proceso y el resultado final. Es por esto, por lo que este proceso se ha realizado de dos maneras diferentes. Se han seleccionado estos tras analizar las posibles hipótesis defensivas que manejan los arqueólogos municipales responsables, a partir de estudios previos.

\subsubsection{Red de anillos concéntricos}

Con esta simulación se pretende obtener la ubicación de la red de atalayas de manera que formen una red de anillos concéntricos respecto a la Fortaleza de la Mota. De esta forma se minimiza el número de comunicaciones a realizar entre atalayas para alertar a la Fortaleza de cualquier incidencia (véase la Figura 9).

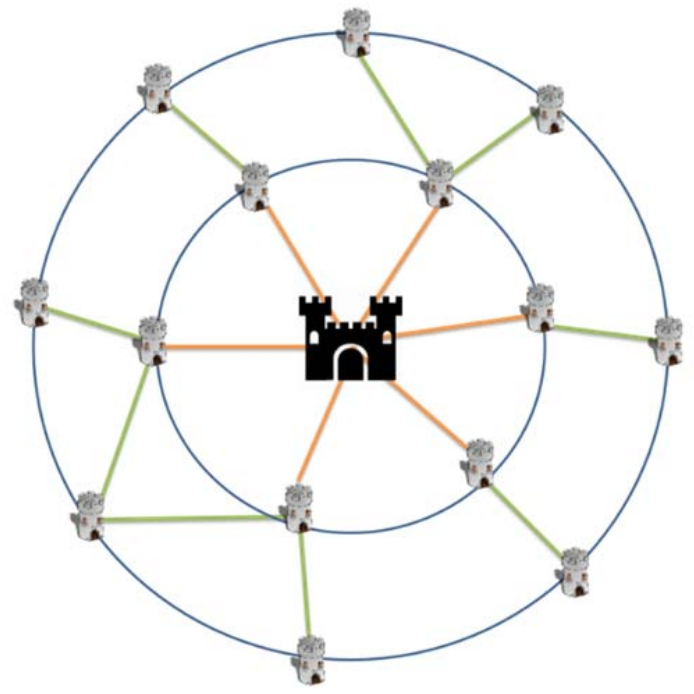

Figura 9: Esquema simulación de red de anillos concéntricos

Así, la Fortaleza tendría comunicación visual con el primer anillo de atalayas y viceversa, pero a su vez el primer anillo tendría comunicación visual con el segundo anillo de atalayas, estableciendo como mínimo una comunicación uno a uno.

\subsubsection{Red ramificada}

Con esta simulación se obtiene la ubicación de la red de atalayas de manera que formen una red lineal, en la que cada elemento se comunique con otro en una relación como mínimo de uno a uno.

Según esta metodología, cada elemento de la red, incluida la Fortaleza, no necesariamente tiene conectividad visual con más de un elemento, de manera que existe menos redundancia de visibilidad entre atalayas (Véase la Figura 10).

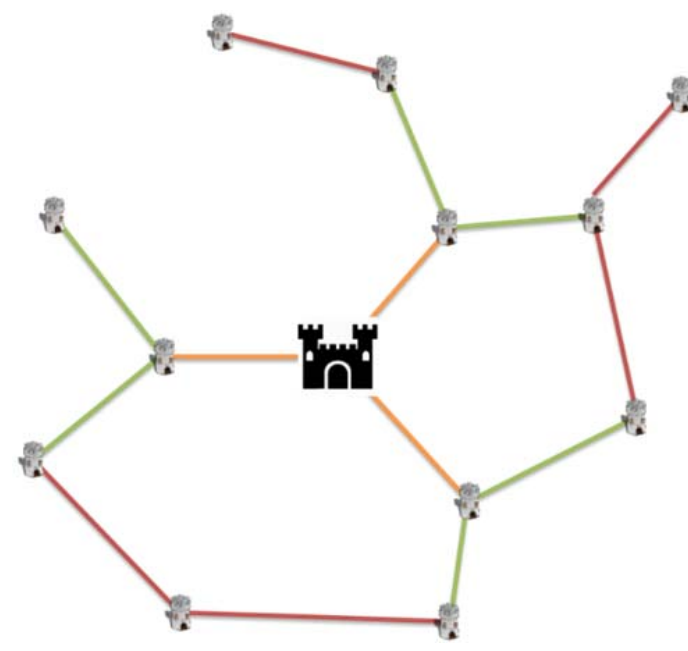

Figura 10: Esquema simulación de red ramificada

\section{Resultados}

\subsection{Determinación de ubicación óptima}

\subsubsection{Simulación 1 - Red de anillos}

A continuación se exponen los resultados obtenidos para la simulación a partir del método de anillos concéntricos. Con esta técnica obtenemos un total de 27 atalayas, 14 situadas en el anillo más cercano a la Fortaleza y 13 en el anillo externo.

A partir de la situación de las atalayas mediante esta simulación y sus respectivas cuencas visuales, se realiza un estudio del comportamiento de la superficie visible por la red (véase la Figura 11).

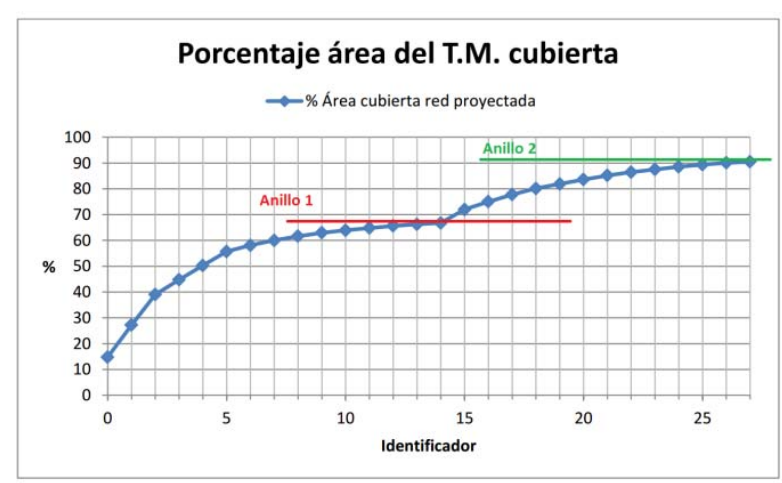

Figura 11: Porcentaje de área visible red de anillos concéntricos

Tal y como se puede ver en la Figura 12, teniendo en cuenta el porcentaje de área visible conseguido, para el primer anillo con la inserción de la torre 14 este porcentaje se estabiliza, de la misma manera que se estabiliza dicho porcentaje en el anillo 2 con la inserción de la torre 27.

\subsubsection{Simulación 2 - Red ramificada}

A continuación se exponen los resultados obtenidos para la simulación a partir del método de red ramificada. Con esta técnica se obtienen un total de 24 atalayas (véase la Figura 13). 


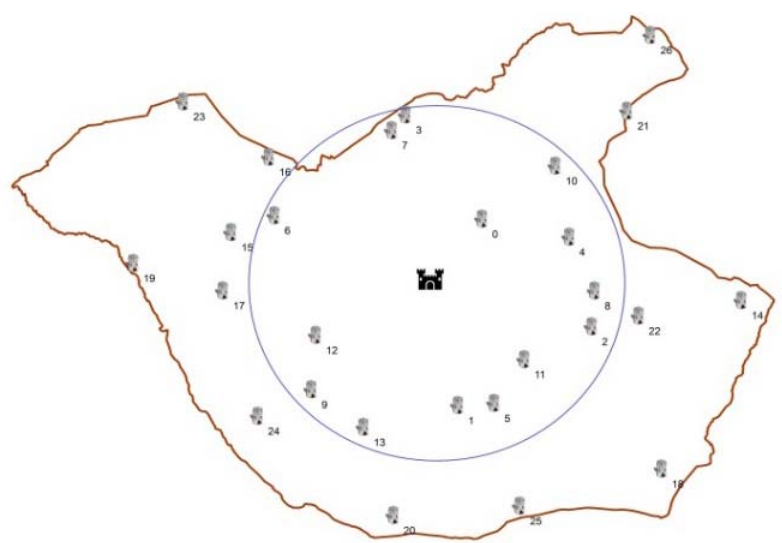

Figura 12: Ubicación atalayas a partir de simulación de red de anillos concéntricos

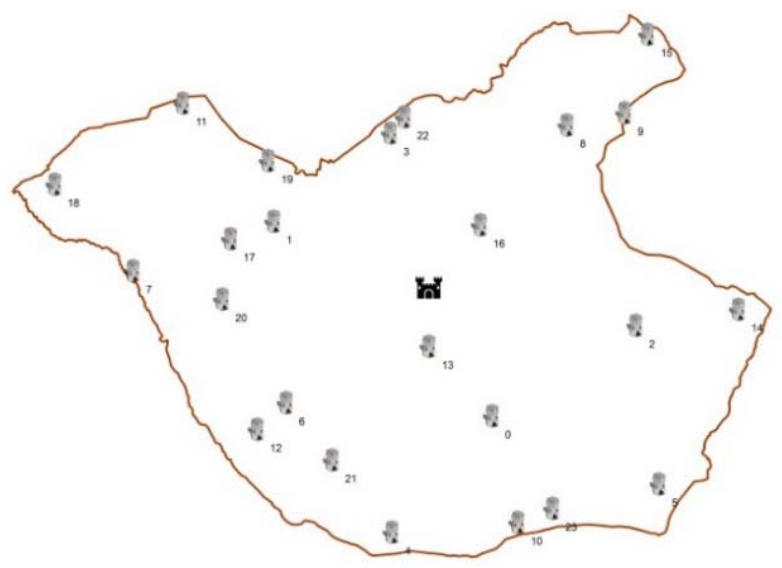

Figura 13: Ubicación atalayas a partir de simulación de red ramificada

A partir de la situación de las atalayas mediante esta simulación y sus respectivas cuencas visuales, se realiza un estudio del comportamiento de la superficie visible por la red.

Tal y como se puede ver en la Figura 14, se observa el comportamiento de la red según esta metodología. El porcentaje de área visible es similar a la conseguida mediante el método de anillos concéntricos pero con un número inferior de elementos.

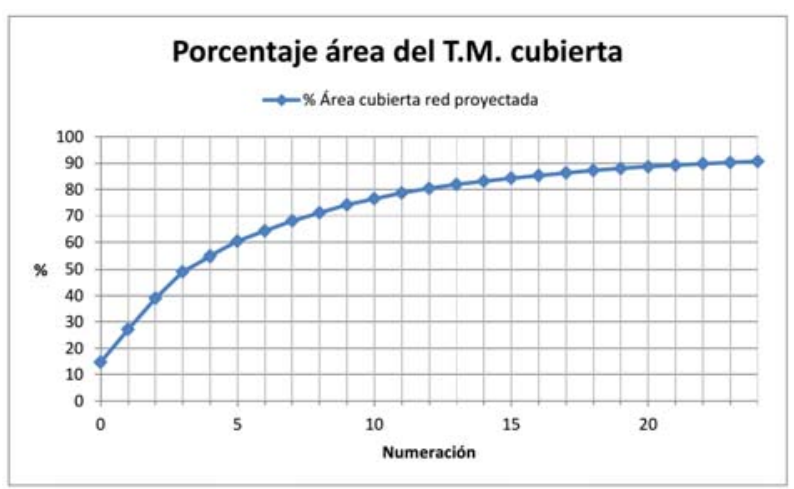

Figura 14: Porcentaje de área visible red ramificada

\subsection{Comparativa entre la red existente y las simuladas}

\subsubsection{Red existente - Red de anillos concéntricos}

A continuación se exponen de manera simultánea la ubicación de las atalayas pertenecientes a la red existente y las obtenidas en la simulación de anillos concéntricos.

Como se puede apreciar en la Figura 15, existen varias coincidencias posicionales entre las atalayas existentes y las simuladas.

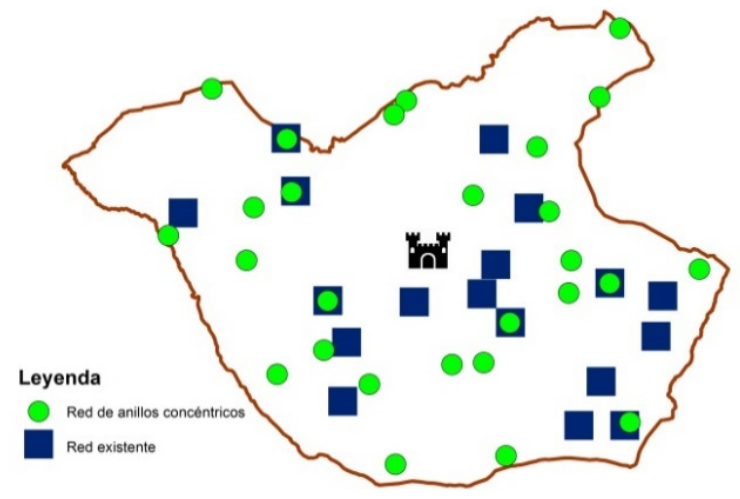

Figura 15: Comparativa entre red existente y red simulada mediante método de red de anillos concéntricos

\subsubsection{Red existente - Red ramificada.}

De la misma manera se exponen superpuestas las ubicaciones de las atalayas pertenecientes a la red existente y las obtenidas en la simulación de red ramificada.

De manera similar a los resultados obtenidos en la simulación de red de anillos concéntricos, existen varias coincidencias posicionales entre atalayas existentes y simuladas (véase la Figura 16).

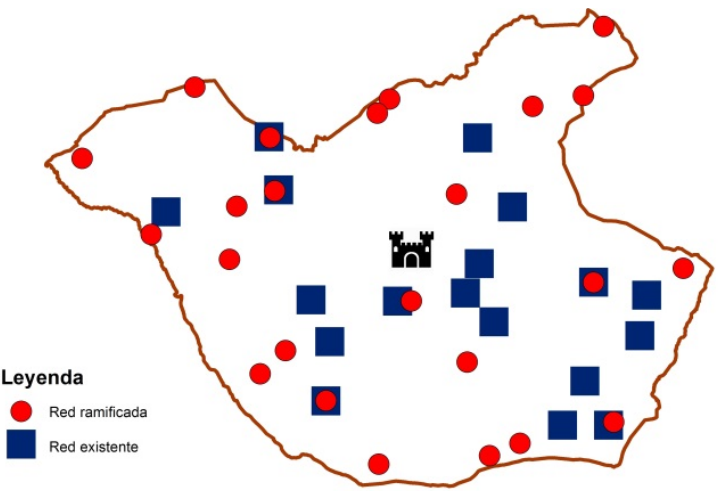

Figura 16: Comparativa entre red existente y red simulada mediante método de red ramificada

\section{Conclusiones}

Destacar el aumento de potencial que se produce utilizando los sistemas de información geográfica y sus herramientas a través de programación, ya que posibilitan la realización de análisis más complejos, además de reutilizar la metodología de la herramienta diseñada en proyectos similares. Los lenguajes de programación nos permiten interactuar con el sistema 
de información geográfica de manera mucho más directa que con la propia interfaz del software.

Por otro lado, la importancia de la fotogrametría en la captura de datos sobre el terreno y para la modelización de monumentos $u$ otros objetos, utilizando imágenes fotográficas, siendo por lo tanto un método totalmente objetivo y con información continua y muy completa.

En cuanto a los resultados obtenidos, destacar la coincidencia posicional entre varias de las atalayas de las redes obtenidas a partir de la metodología propuesta en este trabajo con atalayas de la red existente, teniendo en cuenta la antigüedad de dichas construcciones (algunas de ellas, datan del siglo IX), por lo que es muy probable la desaparición de muchas de ellas, dejando reducida la red de atalayas real. Además de esta circunstancia, hay que tener en cuenta que los límites administrativos actuales sobre los que se ha basado el análisis no corresponden con los de la época en la que se materializaron y utilizaron dichas atalayas.
Finalmente, en cuanto a líneas futuras de trabajo, destacar que el análisis realizado ha tenido en cuenta unos parámetros iniciales muy generales, por lo que el análisis realizado se podría ejecutar de manera más específica. Concretamente, en relación al presente estudio, podrían tenerse en cuenta más parámetros para la simulación como la ubicación de las vías de comunicación, poblaciones colindantes, y diversos elementos que pudiesen afectar en mayor o menor medida a la distribución de las atalayas.

\section{Agradecimientos}

Agradecimientos al departamento Ingeniería Cartográfica, Geodésica y Fotogrametría y al Grupo de Investigación Sistemas Fotogramétricos y Topométricos, por facilitar el material para la realización del presente trabajo.

Agradecer también al Excmo. Ayuntamiento de Alcalá la Real por la aportación de la documentación que disponían acerca del fenómeno de estudio.

\section{Referencias}

MERCHÁN, M.Z., y PREYSLER, J.B., 2012. Los SIG en la Arqueología española: una valoración 'CAA' del contexto actual. Cuadernos de Prehistoria y Arqueología de la Universidad de Granada, 20, 49-64.

OJEDA, E.V., y LORCA, A.M.G., 2012. Comunicación visual mediante técnicas GIS en el litoral del Campo de Dalías: la defensa costera de los pueblos del interior. Virtual Archaeology Review, 3(6), 93-97.

TORRES, J.C., SOLER, F., CANO, P., LEÓN, A., LUZÓN, V., y MARTíN, D., 2012. Sistema de Información para Patrimonio Histórico. Virtual Archaeology Review, 3(5), 53-57. 